\title{
Nanoscale
}

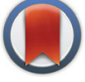

CrossMark \&lick for updates

Cite this: Nanoscale, 2015, 7, 10982

Received 21st February 2015,

Accepted 21st May 2015

DOI: $10.1039 / \mathrm{c} 5 \mathrm{nr} 01204 \mathrm{j}$

www.rsc.org/nanoscale

\section{Dominant luminescence is not due to quantum confinement in molecular-sized silicon carbide nanocrystals $\uparrow$}

\author{
David Beke, ${ }^{\star a, b} Z^{a}$ solt Szekrényes, ${ }^{a}$ Zsolt Czigány, ${ }^{c}$ Katalin Kamarás ${ }^{a}$ and \\ Ádám Gali*a,d
}

\begin{abstract}
Molecular-sized colloid silicon carbide (SiC) nanoparticles are very promising candidates to realize bioinert non-perturbative fluorescent nanoparticles for in vivo bioimaging. Furthermore, SiC nanoparticles with engineered vacancy-related emission centres may realize magneto-optical probes operating at nanoscale resolution. Understanding the nature of molecular-sized SiC nanoparticle emission is essential for further applications. Here we report an efficient and simple method to produce a relatively narrow size distribution of water soluble molecular-sized SiC nanoparticles. The tight control of their size distribution makes it possible to demonstrate a switching mechanism in the luminescence correlated with particle size. We show that molecular-sized SiC nanoparticles of 1-3 nm show a relatively strong and broad surface related luminescence whilst the larger ones exhibit a relatively weak band edge and structural defect luminescence with no evidence of quantum confinement effect.
\end{abstract}

Silicon carbide is a wide bandgap indirect semiconductor ${ }^{1}$ with a variety of applications such as high power electronics due to the high breakdown field, high thermal conductivity, and existence of surface oxide. ${ }^{2}$ Because of the chemical resistivity and high intrinsic temperature $\mathrm{SiC}$ is ideal for applications in harsh environments. ${ }^{3}$ It also exhibits great application potential in ultraviolet (UV) photodiodes, ${ }^{4}$ spintronics ${ }^{5,6}$ and quantum information processing. ${ }^{7-9} \mathrm{SiC}$ can crystallize in several forms called polytypes. These polytypes are identical in two dimensions (hexagonal basal plane) and differ

\footnotetext{
${ }^{a}$ Institute for Solid State Physics and Optics, Wigner Research Centre for Physics, Hungarian Academy of Sciences, PO. Box 49, H-1525 Budapest, Hungary. E-mail:beke.david@wigner.mta.hu,gali.adam@wigner.mta.hu ${ }^{b}$ Faculty of Chemical Technology and Biotechnology, Budapest University of Technology and Economics, Müegyetem rkp. 3., H-1111 Budapest, Hungary ${ }^{c}$ Institute for Technical Physics and Materials Science, Centre for Energy Research, Hungarian Academy of Sciences, Konkoly-Thege M. út 29-33., H-1121 Budapest, Hungary

${ }^{d}$ Department of Atomic Physics, Budapest University of Technology and Economics, Budafoki út 8, H-1111 Budapest, Hungary

$\dagger$ Electronic supplementary information (ESI) available. See DOI: 10.1039/ c5nr01204j
}

in the $\mathrm{Si}-\mathrm{C}$ bilayer sequences in the third dimension ( $c$-axis perpendicular to the basal plane). ${ }^{10}$ As an indirect-bandgap semiconductor, bulk $\mathrm{SiC}$ has weak luminescence, however, porous $\mathrm{SiC}^{11}{ }^{11}$ small nanocrystals ${ }^{12}$ and nanowires ${ }^{13}$ show bright photoluminescence (PL). SiC nanocrystals (NCs) are proven to be favorable biological labels ${ }^{14}$ due to their good biocompatibility, ${ }^{15}$ hemocompatibility ${ }^{16}$ and excellent solubility in polar solvents. ${ }^{17}$ Moreover, they contain many surface groups that are suitable for further chemical modifications and conjugation for targeted biomolecules. ${ }^{18}$ Even though the applicability of SiC NCs in the biological environment ${ }^{19,20}$ and therapy $^{21}$ was demonstrated, understanding the physics behind the luminescence is still in the centre of intense research. In porous $\mathrm{SiC}$ bright luminescence was reported, similar to that in porous $\mathrm{Si}$, but the origin of this luminescence is still unclear. The luminescence of porous $\mathrm{SiC}$ is often associated with the quantum confinement effect, ${ }^{22}$ however, the relatively large crystallite size and the polytype independent luminescence implied that the luminescence was related to surface defects. ${ }^{23}$ Experimental results ${ }^{24,25}$ and theoretical calculations showed that the luminescence of SiC NCs is strongly influenced by the surface groups. ${ }^{26}$ Indeed a SiC NC solution containing $2 \mathrm{~nm}$ nanoparticles shows luminescence with emission at $450 \mathrm{~nm}(2.75 \mathrm{eV})$, nearly independent of excitation wavelength, while calculation showed that hydrogen terminated NCs of this size should emit in the deep UV. ${ }^{27,28} \mathrm{Wu}$ and coworkers gave experimental evidence of quantum confinement in $\mathrm{SiC} \mathrm{NCs}^{29}$ based on the excitation dependent luminescence properties of such NC solutions. This dependence is a necessary but not a sufficient condition to unambiguously prove the quantum confinement effect. There are several reports about excitation dependent luminescence properties of carbon $\operatorname{dots}^{30,31}$ and graphene oxide solutions ${ }^{32}$ where this property is explained by different surface groups and the distribution of these groups. ${ }^{33}$ Guo et al. reported that SiC NCs prepared in ethanol solution possessed low excitation dependent emission in the case of fresh samples but aged samples showed strong excitation dependence. ${ }^{34}$ Their size measurements suggested that SiC NCs aggregated quickly in 
ethanol solution and they associated the changes in the luminescence properties with the change in size distribution of SiC NCs. ${ }^{34}$ These contradicting results create doubt about the simple quantum confinement model and the varying conclusions might come from the different size distribution of the colloid SiC particles.

In this paper we demonstrate an effective size separation method which allows us to prepare a SiC NC solution containing only 1-4 $\mathrm{nm}$ particles. This is an important step toward biomedical and in vivo applications where the hydrodynamic size of the nanocrystals should be less than $5.5 \mathrm{~nm}$ that is needed for clearance. ${ }^{35,36}$ With the separation of small individual SiC NCs from larger or aggregated NCs we show that SiC NCs larger than $4 \mathrm{~nm}$ have different PL properties than those of molecular-sized nanoparticles. We demonstrate that the obtained excitation dependence of the SiC NC solution in previous reports is a convolution of two different emission centres with different PL and photoluminescence excitation (PLE) properties because of the coexistence of molecular-sized 1-4 nm nanoparticles with surface related luminescence and larger nanoparticles or aggregates with band edge (BE) or near band edge (NBE) luminescence.

The recorded PL spectra of as-prepared SiC NCs are shown in Fig. 1. As can be seen the peak maximum at $\sim 450 \mathrm{~nm}$ shifts only $8 \mathrm{~nm}$ upon changing the excitation wavelength between 310 and $370 \mathrm{~nm}$ (marked with a vertical dashed line) but shows a severe reduction in the measured intensity upon excitation with wavelengths longer than $320 \mathrm{~nm}$. We observe another more intense red shift upon excitation with wavelengths of 370-450 nm (marked by the slant dashed line). This shift was previously associated with the quantum confinement effect. ${ }^{29}$ We will demonstrate that this shift is due to the convolution of two different emission centres.

A sample with broad size distribution was centrifuged using a $30 \mathrm{kDa}$ macrosep filter. The remaining solution (sample II) was washed 10 times to remove most of the small particles. It should be noted that the feed was concentrated from $10 \mathrm{ml}$ to $1.5 \mathrm{ml}$ during filtration but not dried. As shown in Fig. 2a, the filtrate (sample I) exhibits a similar peak maximum as the as-prepared sample but the long-wavelength shoulder is missing and there is almost no sign of changing the emission with excitation wavelength.

Fig. $3 \mathrm{a}-\mathrm{c}$ and $\mathrm{d}$ show the size and atomic structure of sample II observed by high transmission resolution spectroscopy (HRTEM) and atomic force microscopy (AFM), respectively, whereas Fig. 3e plots the corresponding PL spectra. The PL spectra of sample II show excitation independent emission with a peak maximum at $530 \mathrm{~nm}(2.39 \mathrm{eV})$. The shape and intensity of the luminescence band are very different from those of sample I.

Despite the wide size distribution no shift occurs in the emission maximum upon changing the excitation wavelength. The $530 \mathrm{~nm}(2.39 \mathrm{eV}) \mathrm{PL}$ signal is in good agreement with the band gap of $3 \mathrm{C}-\mathrm{SiC}(2.35 \mathrm{eV})$. The Bohr radius of $3 \mathrm{C}-\mathrm{SiC}$ is about $2.7 \mathrm{~nm}$ and calculations implied that $4 \mathrm{~nm}$ 3C-SiC NCs have an almost bulk like absorption band. ${ }^{28}$ Based on these

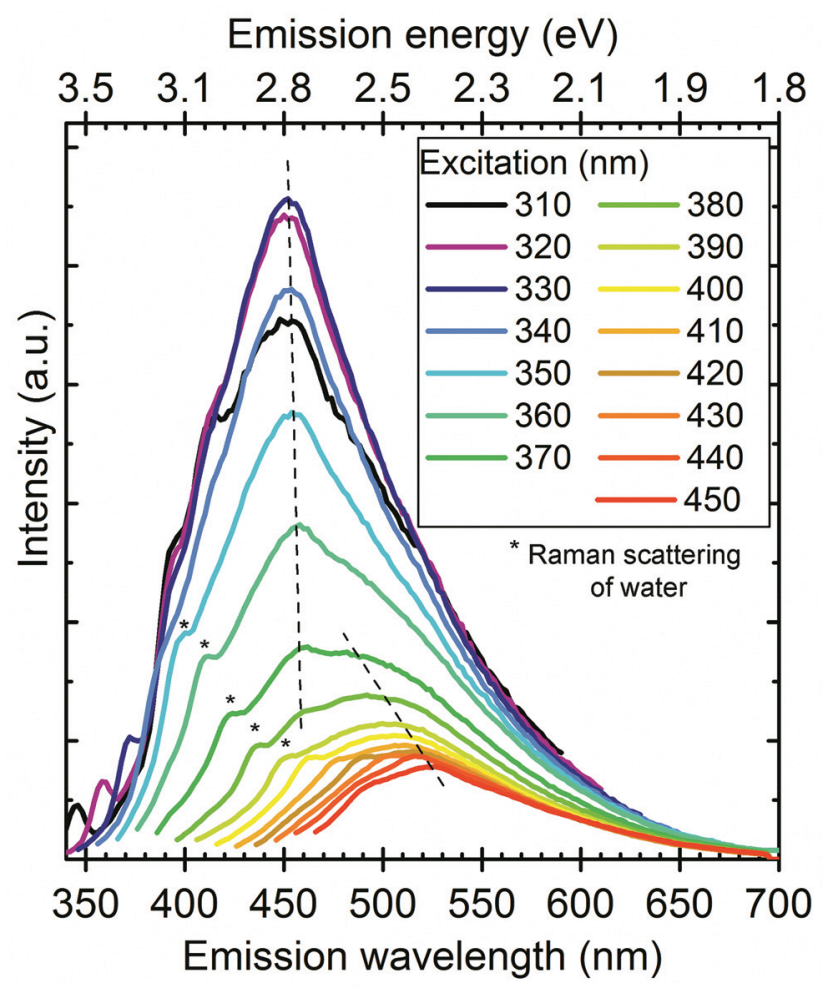

Fig. 1 Photoluminescence spectra of SiC NCs in water at different excitation wavelengths. We attribute this complex spectrum to two types of emission centres represented by dashed lines. An emission centre at $\sim 450 \mathrm{~nm}$ (vertical dashed line) shows a negligible red shift with increasing excitation wavelength whereas another emission centre at the lowfrequency shoulder shows a clear red shift by varying the excitation wavelength between 370 and $450 \mathrm{~nm}$ represented by the slant dashed line. The Raman signal of water is also marked in the spectrum by star symbols.

arguments we attribute the $530 \mathrm{~nm}$ peak to the $\mathrm{BE}$ luminescence of larger particles. While SiC has an indirect band gap, and consequently weak luminescence at room temperature, exciton recombination can be enhanced by the relaxation of selection rules due to the relatively small size of the particles and dielectric confinement. Several additional peaks appear in the PL spectra of sample II at about $408 \mathrm{~nm}(3.02 \mathrm{eV}), 460 \mathrm{~nm}$ $(2.67 \mathrm{eV})$ and $492 \mathrm{~nm}(2.52 \mathrm{eV})$. The first peak may correlate with the BE luminescence of $6 \mathrm{H}$ polytype inclusions ${ }^{37}$ whilst the other two may originate from their stacking faults. ${ }^{38} 6 \mathrm{H}$ inclusion in 3C-SiC may be considered as an "ordered" sequence of stacking faults that can be described as 3-3 zigzag lines consisting of $6 \mathrm{Si}-\mathrm{C}$ bilayers along the $c$-axis (see ESI $\dagger$ ). Stacking faults within the $6 \mathrm{H}$ inclusions embedded in 3C-SiC result in irregular stacking sequences. We show the evidence of such irregular stacking sequences in a larger SiC nanoparticle observed by HRTEM in Fig. 3(a-c). Even though the purchased $\mathrm{SiC}$ is a cubic $3 \mathrm{C}$ powder confirmed by X-ray diffraction measurements before and after etching it (not shown), polytype inclusions are common defects in $\mathrm{SiC}$ and may appear at low concentrations in the $3 \mathrm{C}$-SiC powder. Since the applied etching method works mainly on the cubic 
a

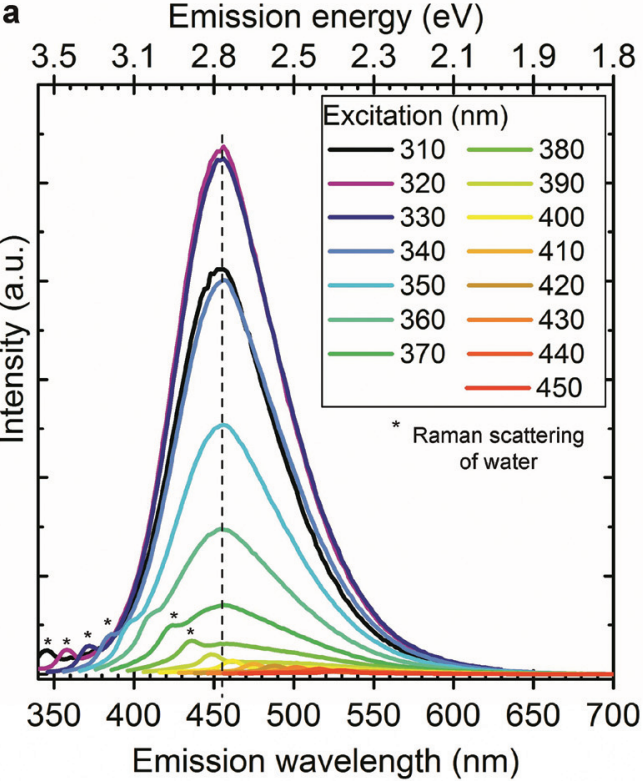

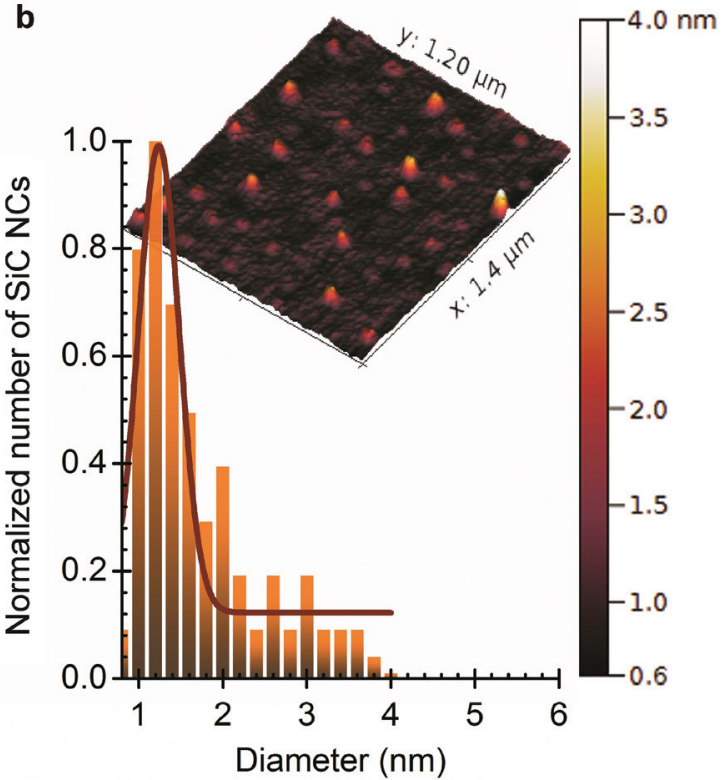

Fig. 2 (a) Shows the PL spectra of sample I (filtrate) at different excitation wavelengths after SiC NCs were filtered through a $30 \mathrm{kDa}$ centrifuge filter. After filtration the red shoulder does not occur in the PL spectra (cf., Fig. 1). (b) Shows the AFM image and size distribution of sample I. The average size is about $1.5 \mathrm{~nm}$ and most of the particles are smaller than $4 \mathrm{~nm}$.

3C-SiC, ${ }^{39}$ the hexagonal polytypes remain mostly intact. Therefore, selective etching of 3C-SiC enhances the concentration of polytype inclusions in our system. While we believe this is the main reason for the detected polytype dispersion, it should be noted that phase transformation was claimed during the preparation of SiC NCs by laser ablation ${ }^{40}$ and also by an etching process similar to ours. ${ }^{34} 6 \mathrm{H}$ inclusions in $3 \mathrm{C}$-SiC enhance the luminescence of $3 \mathrm{C}-\mathrm{SiC}^{41}$ which further explains the detectable $\mathrm{BE}$ luminescence of $3 \mathrm{C}-\mathrm{SiC}$ at room temperature. Fig. 4 shows the photoluminescence excitation (PLE) spectra of sample I and sample II. Sample I has maximum emission efficiency at $320 \mathrm{~nm}$ excitation while the PLE spectrum of sample II corresponds to the PLE of bulk 3C-SiC. ${ }^{42}$ These observations further strengthen our conclusion that the properties of large particles are close to those of bulk 3C-SiC.

Large particles may either form because of the repeated etching of SiC powder reducing the size of the starting materials or may come from the aggregation of smaller particles. To unravel the role of either of these mechanisms in the change of the luminescence band we dried a droplet of sample I on the Si surface consisting of only molecular-sized nanoparticles and measured the PL during drying. Fig. 5 shows how the PL changed during water evaporation. First, SiC NCs are surrounded with strongly adsorbed water molecules, ${ }^{24}$ thus the PL of this sample is almost identical to the PL of SiC NCs in solution. As we successively remove the hydration shell around the $\mathrm{SiC}$ nanoparticles, the emission maximum shifts to lower wavelengths typical of the PL signal of sample II. We conclude from the change in the PL bands that water evaporation is a two-step process: first the disappearance of the excess water, leaving the hydrated nanoparticles intact, followed by the disruption of the hydration shells and the subsequent forming of aggregates which leads to the PL bands characteristic of sample II. This result indicates that sample II may contain aggregates.

Aggregated or closely packed NCs usually have different optical properties from those of the individual particles. ${ }^{43,44}$ It should be noted that even though surfactants are not used to stabilize our SiC NCs because of the high colloid stability of the particles, recrystallization or Ostwald ripening effect is not probable because of the high stability of the $\mathrm{Si}-\mathrm{C}$ bond. The wavefunctions in two closely lying SiC NCs may overlap building up crystalline bands ${ }^{43,45}$ that can lead to bulk-like optical properties. This effect is known in similar nanoparticle systems. ${ }^{4-45}$

We did not find any sign of size dependent optical properties in the 3C-SiC colloid system, however, the size distribution was relatively broad in both parts of the separated samples. SiC NCs made by electroless wet chemical etching contain a large number of oxidized surface groups because of the applied strong acids. We conclude that SiC NCs could be rather described as $\mathrm{Si}_{x} \mathrm{C}_{y} \mathrm{O}_{z}(\mathrm{H})$ systems. In the case of molecular-sized SiC NCs surface related luminescence is dominant. ${ }^{34,46}$ The surface related luminescence originates from localized states that have weak NC size dependency. ${ }^{47}$ However, various oxygen-containing surface groups may contribute to the PL spectrum depending on the surface environment possessing considerable Stokes shifts ${ }^{24,26,27}$ that result in a relatively broad PL signal at room temperature. As the particles become larger, the surface to volume ratio becomes small and the oxygen content becomes negligible. Our results 

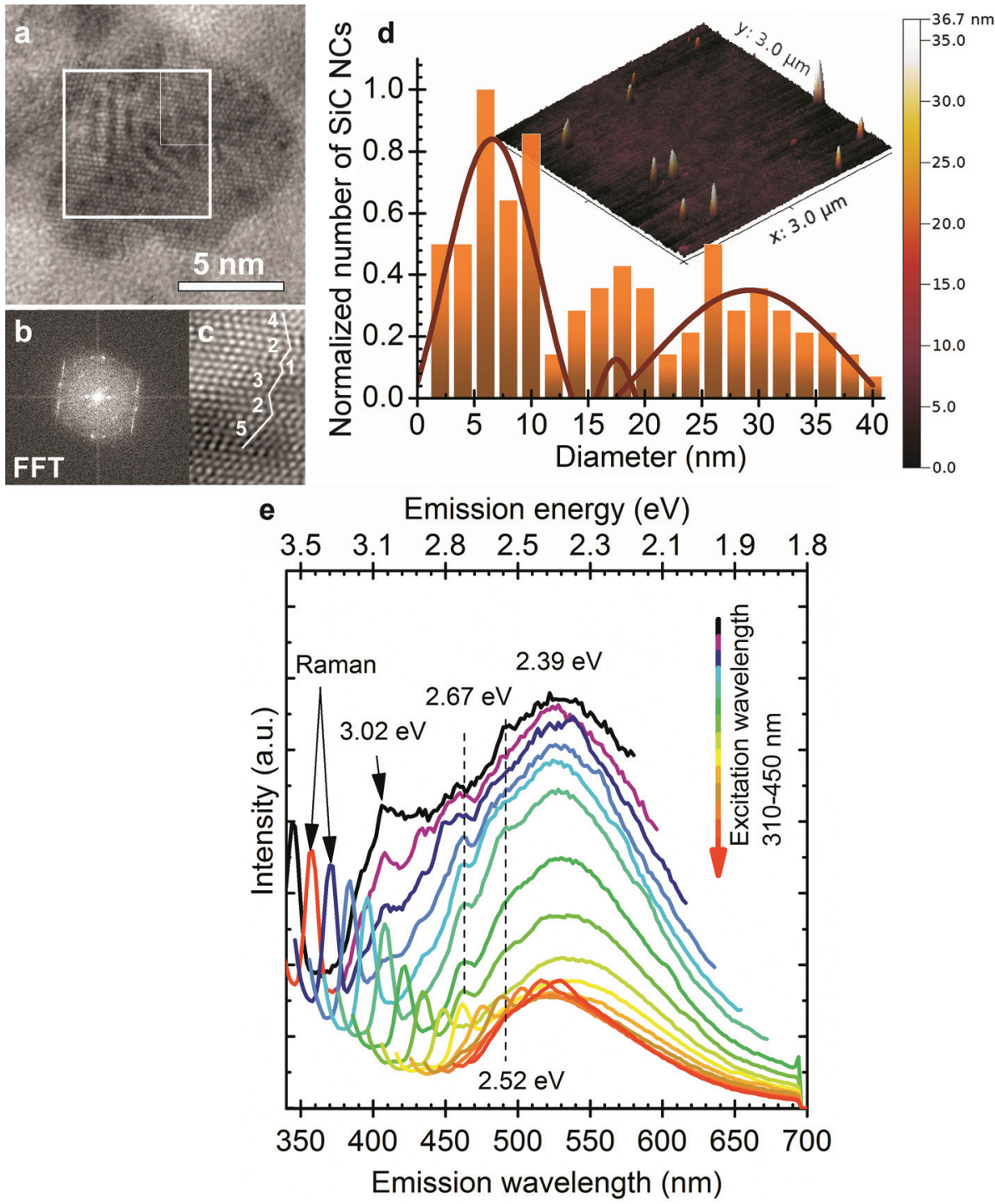

Fig. 3 (a) HRTEM image of a $\sim 10 \mathrm{~nm} \mathrm{SiC} \mathrm{nanoparticle.} \mathrm{(b)} \mathrm{Fast} \mathrm{Fourier} \mathrm{transform} \mathrm{(FFT)} \mathrm{of} \mathrm{the} \mathrm{area} \mathrm{marked} \mathrm{with} \mathrm{a} \mathrm{square} \mathrm{in} \mathrm{panel} \mathrm{(a).} \mathrm{(c)} \mathrm{Fourier}$ filtered HRTEM image of the area marked with a rectangle in the upper right corner of the square in panel (a). An irregular stacking sequence of 5-23-1-2-4 is indicated in the figure. See text for more explanation. (d) shows AFM image and the size distribution of sample II. Sample II contains particles with size between 2 and $40 \mathrm{~nm} .3$ Gauss functions can be fitted to the size distribution with maxima at $6.5 \mathrm{~nm}, 17.5 \mathrm{~nm}$ and $29.0 \mathrm{~nm}$. (e) PL spectra of sample II (filtrand) at different excitation wavelengths after SiC NCs were filtered through a $30 \mathrm{kDa}$ centrifuge filter. The broad peak at $530 \mathrm{~nm}(2.39 \mathrm{eV})$ is the BE luminescence of SiC while peaks at $408 \mathrm{~nm}(3.02 \mathrm{eV}), 464 \mathrm{~nm}(2.67 \mathrm{eV})$ and $492 \mathrm{~nm}(2.52 \mathrm{eV})$ are associated with the BE of $6 \mathrm{H}$ polytype inclusions (sequence of 3 in c) and their stacking faults (sequences other than 3 in c). The Raman peaks of the absorbed water are also indicated ('Raman'); they have comparable intensity to that of the detected PL of SiC particles.

confirm the conclusion of theoretical calculations stating ${ }^{27,46}$ that the core recombination becomes dominant for nanoparticles with sizes of $4 \mathrm{~nm}$ and above while surface related luminescence dominates in smaller SiC nanoparticles.

In conclusion, we demonstrated an effective separation of molecular-sized bioinert SiC nanoparticles from larger aggregates in colloid SiC NC solution. These two fractions possess significantly different PL signals. PL is proven to be a very simple and efficient tool to verify the presence of larger aggregates in the colloid samples using a PL peak at around $530 \mathrm{~nm}$. Our results show that the molecular-sized SiC NCs indeed exhibit surface group-related broad luminescence between 400 and $600 \mathrm{~nm}$ with a maximum at $450 \mathrm{~nm}$. This broad luminescence may play an important role in the context of magneto-optical color centres in nanocrystalline $\mathrm{SiC}^{47,48} \mathrm{It}$ has been proposed that molecular-sized SiC NCs embedding paramagnetic color centres may be ultimate fluorescent biomarkers that might be used even for quantum metrology going beyond the traditional dyes. ${ }^{14}$ We demonstrated here that molecular-sized SiC NCs themselves possess complex emission properties. We note that the fluorescence of color centres introduced in these SiC NCs might be compromised by the 


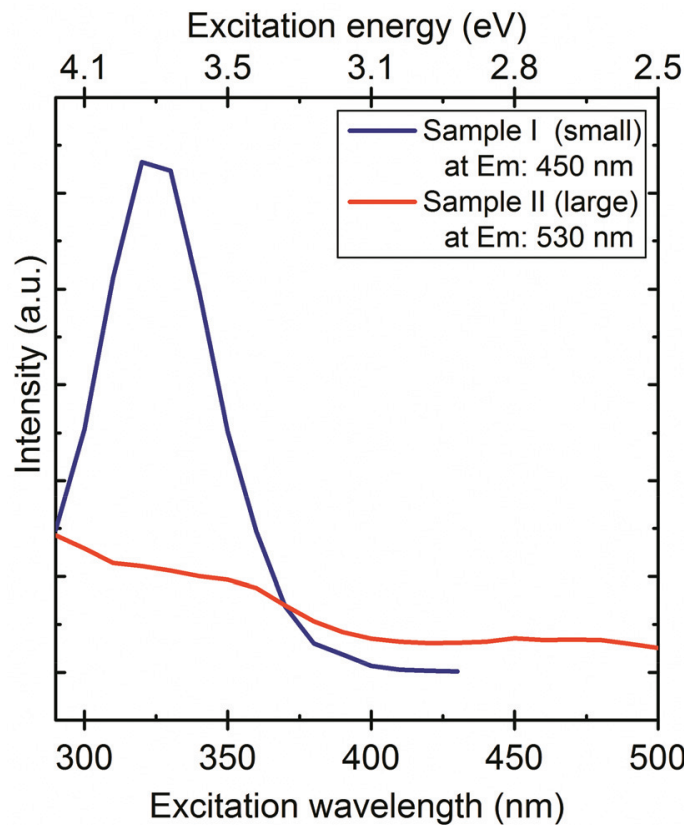

Fig. 4 PLE spectra of SiC NCs with different size distribution where the emission wavelength ("at Em:") is indicated. Sample I contains 1-4 nm particles, sample II contains particles larger than $5 \mathrm{~nm}$. The different line shapes confirm that different absorption and emission mechanisms take place in these two systems.

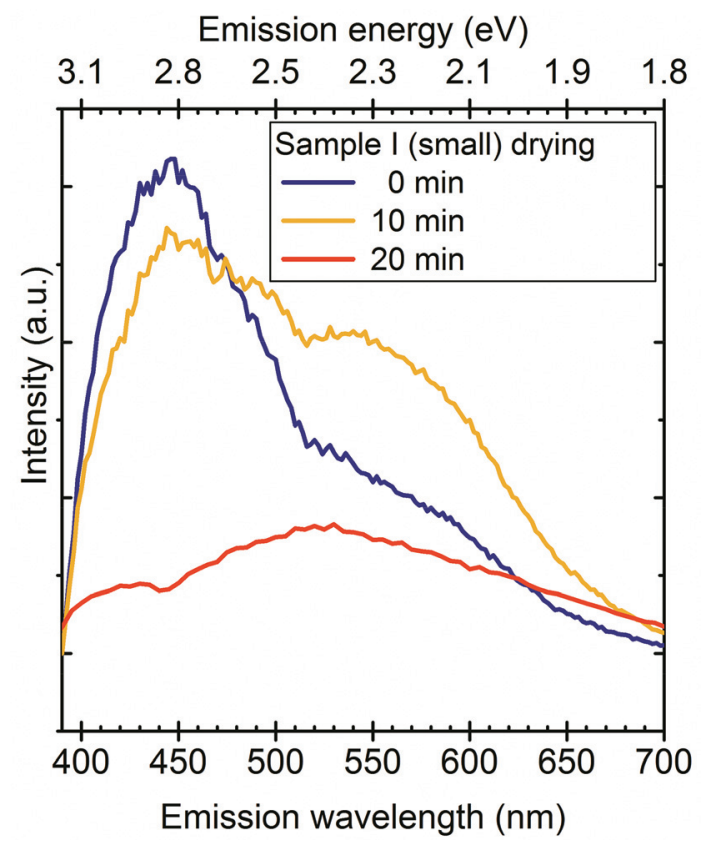

Fig. 5 PL spectra of SiC NC solution containing small particles during drying on the Si surface. The emission is changing due to water evaporation and aggregation. The measurement was carried out by heating the sample on a $100{ }^{\circ} \mathrm{C}$ hot plate and placing it into the spectrometer at 5 minute intervals. surface groups responsible for the emission of SiC NCs which is a subject of studies in the near future.

\section{Experimental section}

The 3C-SiC powder with particle sizes of about 1-10 $\mu \mathrm{m}$ (US Research Nanomaterials Inc.) was etched in a $\mathrm{HF}: \mathrm{HNO}_{3}$ solution for the preparation of SiC NCs. The synthesis method was reported elsewhere. ${ }^{49}$ Briefly, SiC powder was placed in an acid digestion chamber with concentrated $\mathrm{HF}: \mathrm{HNO}_{3}$ solution and etched for 2 hours at $100{ }^{\circ} \mathrm{C}$. During the etching a thin porous layer is formed on the surface of the particles. After the removal of the acid and sonication of the porous $\mathrm{SiC}$ we obtain 1-5 nm SiC NCs and large particle residues that can be removed by centrifugation. Repeating the etching process on the microparticles, they shrink slowly and the size distribution of the nanoparticles becomes wider. The samples were studied by photoluminescence (PL; Horiba Jobin Yvon NanoLog), atomic force microscopy (AFM; NeaSpec), and high resolution transmission electron microscopy (HRTEM; JEOL JEM-3010). The PL was measured with a $450 \mathrm{~W}$ Xe lamp and $3 \mathrm{~nm}$ bandwidth for SiC colloid solution unless noted. We applied a standard spin coating technique to separate the nanoparticles dried on a silicon substrate, in order to study them by AFM. For size distribution measurements, about 300 particles were measured with AFM on different places of the substrate. We deposited the dried SiC particles on a thin carbon layer for HRTEM study and about 300 particles were analyzed.

\section{Acknowledgements}

A.G. acknowledges the funding support from the Hungarian OTKA Grant No. 101819 and 106114, and the MTA Lendület programme from the Hungarian Academy of Sciences. K.K. and Zs.Sz. acknowledge the funding support from the Hungarian OTKA Grant no. 105691.

\section{References}

1 G. Rosario, Properties and Applications of Silicon Carbide, InTech, 2011, ISBN 978-953-307-201-2.

2 J. W. Palmour, J. A. Edmond, H. S. Kong and C. H. Carter, 6H-Silicon Carbide Devices and Applications, Phys. Rev. B: Condens. Matter, 1993, 185, 461-465.

3 M. Mehregany, SiC MEMS: Opportunities and Challenges for Applications in Harsh Environments, Thin Solid Films, 1999, 355-356, 518-524.

4 A. Powell, Growth Of Sic Substrates, Int. J. High Speed Electron. Syst., 2006, 16, 751.

5 W. F. Koehl, B. B. Buckley, F. J. Heremans, G. Calusine and D. D. Awschalom, Room Temperature Coherent Control of Defect Spin Qubits in Silicon Carbide, Nature, 2011, 479, 84-87. 
6 A. L. Falk, P. V. Klimov, B. B. Buckley, V. Ivády, I. A. Abrikosov, G. Calusine, W. F. Koehl, Á. Gali and D. D. Awschalom, Electrically and Mechanically Tunable Electron Spins in Silicon Carbide Color Centers, Phys. Rev. Lett., 2014, 112, 187601.

7 S. Castelletto, B. C. Johnson, V. Ivády, N. Stavrias, T. Umeda, A. Gali and T. Ohshima, A Silicon Carbide Room-Temperature Single-Photon Source, Nat. Mater., 2014, 13, 151-156.

8 M. Widmann, S.-Y. Lee, T. Rendler, N. T. Son, H. Fedder, S. Paik, L.-P. Yang, N. Zhao, S. Yang, I. Booker, et al. Coherent Control of Single Spins in Silicon Carbide at Room Temperature, Nat. Mater., 2015, 14, 164-168.

9 D. J. Christle, A. L. Falk, P. Andrich, P. V. Klimov, J. U. Hassan, N. T. Son, E. Janzén, T. Ohshima and D. D. Awschalom, Isolated Electron Spins in Silicon Carbide with Millisecond-Coherence Times, Nat. Mater., 2015, 14, 160-163.

10 H. Morkoç, S. Strite, G. B. Gao, M. E. Lin, B. Sverdlov and M. Burns, Large-Band-Gap SiC, III-V Nitride, and II-VI ZnSe-Based Semiconductor Device Technologies, J. Appl. Phys., 1994, 76, 1363.

11 W. Shin, W. Seo, O. Takai and K. Koumoto, Surface Chemistry of Porous Silicon Carbide, J. Electron. Mater., 1998, 27, 304-307.

12 M. Mwania, C. Janáky, K. Rajeshwar and P. Kroll, Fabrication of B-SiC Quantum Dots by Photo-Assisted Electrochemical Corrosion of Bulk Powders, Electrochem. Commun., 2013, 37, 1-4.

$13 \mathrm{~J}$. Fan, X. Wu and P. Chu, Low-Dimensional SiC Nanostructures: Fabrication, Luminescence, and Electrical Properties, Prog. Mater. Sci., 2006, 51, 983-1031.

14 B. Somogyi and A. Gali, Computational Design of in Vivo Biomarkers, J. Phys.: Condens. Matter, 2014, 26, 143202.

15 S. Barillet, M.-L. Jugan, M. Laye, Y. Leconte, N. HerlinBoime, C. Reynaud and M. Carrière, In Vitro Evaluation of SiC Nanoparticles Impact on A549 Pulmonary Cells: Cyto-, Genotoxicity and Oxidative Stress, Toxicol. Lett., 2010, 198, 324-330.

16 N. Schettini, M. J. Jaroszeski, L. West and S. E. Saddow, Hemocompatibility Assessment of 3C-SiC for Cardiovascular Applications in Silicon Carbide Biotechnology, Elsevier Inc., 1st edn, 2012, Chapter 5, pp. 153-208, ISBN: 978-0-12385906-8.

17 J. Fan, X. Wu, P. Zhao and P. Chu, Stability of Luminescent 3C-SiC Nanocrystallites in Aqueous Solution, Phys. Lett. A, 2006, 360, 336-338.

18 D. Beke, Z. Szekrényes, I. Balogh, M. Veres, E. Fazakas, L. K. Varga, K. Kamarás, Z. Czigány and A. Gali, Characterization of Luminescent Silicon Carbide Nanocrystals Prepared by Reactive Bonding and Subsequent Wet Chemical Etching, Appl. Phys. Lett., 2011, 99, 213108.

19 D. Beke, Z. Szekrényes, D. Pálfi, G. Róna, I. Balogh, P. A. Maák, G. Katona, Z. Czigány, K. Kamarás, B. Rózsa, et al. Silicon Carbide Quantum Dots for Bioimaging, J. Mater. Res., 2013, 28, 205-209.
20 J. Botsoa, V. Lysenko, a. Géloën, O. Marty, J. M. Bluet and G. Guillot, Application of 3C-SiC Quantum Dots for Living Cell Imaging, Appl. Phys. Lett., 2008, 92, 173902.

21 B. Mognetti, A. Barberis, S. Marino, F. Di Carlo, V. Lysenko, O. Marty and A. Géloën, Preferential Killing of Cancer Cells Using Silicon Carbide Quantum Dots, J. Nanosci. Nanotechnol., 2010, 10, 7971-7975.

22 T. Matsumoto, J. Takahashi, T. Tamaki, T. Futagi, H. Mimura and Y. Kanemitsu, Blue-Green Luminescence from Porous Silicon Carbide, Appl. Phys. Lett., 1994, 64, 226.

23 T. L. Rittenhouse, Surface-State Origin for the Blueshifted Emission in Anodically Etched Porous Silicon Carbide, J. Appl. Phys., 2004, 95, 490.

24 Z. Szekrényes, B. Somogyi, D. Beke, G. Károlyházy, I. Balogh, K. Kamarás and A. Gali, Chemical Transformation of Carboxyl Groups on the Surface of Silicon Carbide Quantum Dots, J. Phys. Chem. C, 2014, 118, 1999520001.

25 D. Dai, X. Guo and J. Fan, Identification of Luminescent Surface Defect in SiC Quantum Dots, Appl. Phys. Lett., 2015, 106, 053115.

26 M. Vörös, P. Deák, T. Frauenheim and A. Gali, The Absorption of Oxygenated Silicon Carbide Nanoparticles, J. Chem. Phys., 2010, 133, 064705.

27 M. Vörös, P. Deák and T. Frauenheim, Time-Dependent Density Functional Calculations on Hydrogenated Silicon Carbide Nanocrystals, Mater. Sci. Forum, 2011, 679-680, 516-519.

28 M. Vörös, P. Deák, T. Frauenheim and A. Gali, The Absorption Spectrum of Hydrogenated Silicon Carbide Nanocrystals from Ab Initio Calculations, Appl. Phys. Lett., 2010, 96, 051909.

29 X. Wu, J. Fan, T. Qiu, X. Yang, G. Siu and P. Chu, Experimental Evidence for the Quantum Confinement Effect in 3C-SiC Nanocrystallites, Phys. Rev. Lett., 2005, 94, 026102.

30 S. K. Bhunia, A. Saha, A. R. Maity, S. C. Ray and N. R. Jana, Carbon Nanoparticle-Based Fluorescent Bioimaging Probes, Sci. Rep., 2013, 3, 1473.

31 S. Chandra, S. H. Pathan, S. Mitra, B. H. Modha, A. Goswami and P. Pramanik, Tuning of Photoluminescence on Different Surface Functionalized Carbon Quantum Dots, RSC Adv., 2012, 2, 3602-3606.

32 Y. Dong, J. Shao, C. Chen, H. Li, R. Wang, Y. Chi, X. Lin and G. Chen, Blue Luminescent Graphene Quantum Dots and Graphene Oxide Prepared by Tuning the Carbonization Degree of Citric Acid, Carbon, 2012, 50, 47384743.

33 S. Zhu, Y. Song, X. Zhao, J. Shao, J. Zhang and B. Yang, The Photoluminescence Mechanism in Carbon Dots (graphene Quantum Dots, Carbon Nanodots and Polymer Dots): Current State and Future Perspective, Nano Res., 2015, 8, 355-381.

34 X. Guo, D. Dai, B. Fan and J. Fan, Experimental Evidence of $\mathrm{A} \rightarrow \mathrm{B}$ Phase Transformation in SiC Quantum Dots and 
Their Size-Dependent Luminescence, Appl. Phys. Lett., 2014, 105, 193110.

35 H. S. Choi, W. Liu, P. Misra, E. Tanaka, J. P. Zimmer, I. B. Ipe, M. G. Bawendi and J. V. Frangioni, Renal Clearance of Quantum Dots, Nat. Biotechnol., 2007, 25, 11651170.

36 X. D. Zhang, J. Yang, S. S. Song, W. Long, J. Chen, X. Shen, H. Wang, Y. M. Sun, P. X. Liu and S. Fan, Passing through the Renal Clearance Barrier: Toward Ultrasmall Sizes with Stable Ligands for Potential Clinical Applications, Int. J. Nanomed., 2014, 9, 2069-2072.

37 G. Wei, W. Qin, G. Wang, J. Sun, J. Lin, R. Kim, D. Zhang and K. Zheng, The Synthesis and Ultraviolet Photoluminescence of $6 \mathrm{H}-\mathrm{SiC}$ Nanowires by Microwave Method, J. Phys. D: Appl. Phys., 2008, 41, 235102.

38 G. Emelchenko, A. Zhokhov, I. I. Tartakovskii, A. Maksimov and E. Steinman, On Peculiarities of Defect Formation in 6H-SiC Bulk Single Crystals Grown by PVT Method, Mater. Sci. Forum, 2013, 740-742, 43-47.

39 G. Z. Cambaz, G. N. Yushin, Y. Gogotsi and V. G. Lutsenko, Anisotropic Etching of SiC Whiskers, Nano Lett., 2006, 6, 548-551.

40 J. Zhu, S. Hu, W. Xia, T. Li, L. Fan and H. Chen, Photoluminescence of $\sim 2 \mathrm{~nm}$ 3C-SiC Quantum Dots Fabricated from Polycrystalline $6 \mathrm{H}-\mathrm{SiC}$ Target by Pulsed Laser Ablation, Mater. Lett., 2014, 132, 210-213.

41 S. I. Vlaskina, G. N. Mishinova, V. I. Vlaskin, V. E. Rodionov and G. S. Svechnikov, 3C-6H Transformation in Heated Cubic Silicon Carbide 3C-SiC, Semicond. Physics, Quantum Electron. Optoelectron., 2011, 14, 432-436.

42 A. A. Gippius, R. Helbig and J. P. F. Sellschop, SiC, Natural and Synthetic Diamond and Related Materials, Elsevier B.V.
North-holland, Amsterdam, 1st edn, 1991, ISBN: 9780444596772.

43 C. Kagan, C. Murray and M. Bawendi, Long-Range Resonance Transfer of Electronic Excitations in Close-Packed CdSe Quantum-Dot Solids, Phys. Rev. B: Condens. Matter, 1996, 54, 8633-8643.

44 O. I. Mićić, S. P. Ahrenkiel and A. J. Nozik, Synthesis of Extremely Small InP Quantum Dots and Electronic Coupling in Their Disordered Solid Films, Appl. Phys. Lett., 2001, 78, 4022 .

45 S. K. Sarkar and G. Hodes, Charge Overlap Interaction in Quantum Dot Films: Time Dependence and Suppression by Cyanide Adsorption, J. Phys. Chem. B, 2005, 109, 72147219.

46 S. Yang, B. Kiraly, W. Y. Wang, S. Shang, B. Cao, H. Zeng, Y. Zhao, W. Li, Z.-K. Liu, W. Cai, et al. Fabrication and Characterization of Beaded SiC Quantum Rings with Anomalous Red Spectral Shift, Adv. Mater., 2012, 24, 55985603.

47 B. Somogyi, V. Zólyomi and A. Gali, Near-Infrared Luminescent Cubic Silicon Carbide Nanocrystals for in Vivo Biomarker Applications: An Ab Initio Study, Nanoscale, 2012, 4, 7720-7726.

48 A. Krueger, G. V. Astakhov, A. Muzha, F. Fuchs, N. V. Tarakina, D. Simin, M. Trupke and V. A. Soltamov, Room-Temperature near-Infrared Silicon Carbide Nanocrystalline Emitters Based on Optically Aligned Spin Defects, Appl. Phys. Lett., 2014, 105, 243112.

49 D. Beke, Z. Szekrényes, I. Balogh, Z. Czigány, K. Kamarás and A. Gali, Preparation of Small Silicon Carbide Quantum Dots by Wet Chemical Etching, J. Mater. Res., 2013, 28, 44-49. 